This is an electronic reprint of the original article. This reprint may differ from the original in pagination and typographic detail.

Author(s): Uimonen, Anna-Maija; Stefanucci, G.; van Leeuwen, Robert

Title: $\quad$ Ultra-nonlocality in density functional theory for photo-emission spectroscopy

Year: $\quad 2014$

Version:

Please cite the original version:

Uimonen, A.-M., Stefanucci, G., \& van Leeuwen, R. (2014). Ultra-nonlocality in density functional theory for photo-emission spectroscopy. Journal of Chemical Physics, 140(18), Article 18A526. https://doi.org/10.1063/1.4868114

All material supplied via JYX is protected by copyright and other intellectual property rights, and duplication or sale of all or part of any of the repository collections is not permitted, except that material may be duplicated by you for your research use or educational purposes in electronic or print form. You must obtain permission for any other use. Electronic or print copies may not be offered, whether for sale or otherwise to anyone who is not an authorised user. 


\section{AD| $\begin{aligned} & \text { The Journal of } \\ & \text { Chemical Physics }\end{aligned}$}

\section{Ultra-nonlocality in density functional theory for photo-emission spectroscopy}

A.-M. Uimonen, G. Stefanucci, and R. van Leeuwen

Citation: The Journal of Chemical Physics 140, 18 A526 (2014); doi: 10.1063/1.4868114

View online: http://dx.doi.org/10.1063/1.4868114

View Table of Contents: http://scitation.aip.org/content/aip/journal/jcp/140/18?ver=pdfcov

Published by the AIP Publishing

\section{Articles you may be interested in}

Analytic cubic and quartic force fields using density-functional theory

J. Chem. Phys. 140, 034103 (2014); 10.1063/1.4861003

Design of effective kernels for spectroscopy and molecular transport: Time-dependent current-density-functional theory

J. Chem. Phys. 134, 084102 (2011); 10.1063/1.3558738

Time-dependent density functional theory of open quantum systems in the linear-response regime

J. Chem. Phys. 134, 074116 (2011); 10.1063/1.3549816

Double-hybrid density-functional theory made rigorous

J. Chem. Phys. 134, 064113 (2011); 10.1063/1.3544215

Magnetic exchange couplings from noncollinear spin density functional perturbation theory

J. Chem. Phys. 129, 194107 (2008); 10.1063/1.3013602

\section{A|P| $\left.\right|_{\text {Applied Physics }} ^{\text {Journal of }}$}

Journal of Applied Physics is pleased to announce André Anders as its new Editor-in-Chief 


\title{
Ultra-nonlocality in density functional theory for photo-emission spectroscopy
}

\author{
A.-M. Uimonen, ${ }^{1}$ G. Stefanucci, ${ }^{2,3,4}$ and R. van Leeuwen ${ }^{1,4}$ \\ ${ }^{1}$ Department of Physics, Nanoscience Center, University of Jyväskylä, Survontie 9, 40014 Jyväskylä, Finland \\ ${ }^{2}$ Dipartimento di Fisica, Universitá di Roma Tor Vergata, Via della Ricerca Scientifica, 00133 Rome, Italy \\ ${ }^{3}$ INFN, Laboratori Nazionali di Frascati, Via E. Fermi 40, 00044 Frascati, Italy \\ ${ }^{4}$ European Theoretical Spectroscopy Facility (ETSF), Louvain-la Neuve, Belgium
}

(Received 26 November 2013; accepted 27 February 2014; published online 19 March 2014)

\begin{abstract}
We derive an exact expression for the photocurrent of photo-emission spectroscopy using timedependent current density functional theory (TDCDFT). This expression is given as an integral over the Kohn-Sham spectral function renormalized by effective potentials that depend on the exchange-correlation kernel of current density functional theory. We analyze in detail the physical content of this expression by making a connection between the density-functional expression and the diagrammatic expansion of the photocurrent within many-body perturbation theory. We further demonstrate that the density functional expression does not provide us with information on the kinetic energy distribution of the photo-electrons. Such information can, in principle, be obtained from TDCDFT by exactly modeling the experiment in which the photocurrent is split into energy contributions by means of an external electromagnetic field outside the sample, as is done in standard detectors. We find, however, that this procedure produces very nonlocal correlations between the exchange-correlation fields in the sample and the detector. () 2014 AIP Publishing LLC. [http://dx.doi.org/10.1063/1.4868114]
\end{abstract}

\section{INTRODUCTION}

The photo-electric effect, in which electrons are emitted from a material by applying light, has played an important conceptual role in quantum mechanics. Already in 1905, Einstein $^{1}$ established the famous relation

$$
E_{\mathrm{K}}=\hbar \omega-\Phi,
$$

where $E_{\mathrm{K}}$ is the maximum kinetic energy of the emitted photo-electrons, $\hbar \omega$ the energy of the incoming photons and $\Phi$ the work function of the material (which is equal to minus the chemical potential provided we use a gauge in which the potential is zero at infinity ${ }^{2}$ ). Presently, photo-emission spectroscopy is a well-developed tool for the study of bandstructures and surface properties of materials (for a review see, e.g., Ref. 3) in which, apart from the kinetic energy, also the angular distribution of the photo-electrons is measured. The photo-emission spectrum is closely related to the spectral function of the material which can exhibit a wide range of many-body features such as quasi-particle broadening and plasmon satellites. Furthermore, there are so-called extrinsic effects describing the energy losses of the photoelectron within the material on its way to the surface. The proper treatment of all these phenomena requires a manybody description. The underlying theory has been described in a number of classic references. ${ }^{4-7}$ Although these manybody approaches can deal with complex many-body processes they are computationally expensive. One may therefore wonder whether one could develop a computationally more efficient approach based on density-functional theory. ${ }^{8-11}$ As the photo-emission process is a time-dependent phenomenon we need a time-dependent version of density functional theory. ${ }^{11-15}$ Since the outgoing photocurrent density $\mathbf{j}(\mathbf{r}, t)$ is a key variable in time-dependent current-density functional theory (TDCDFT $)^{11,16-18}$ an approach based on this formalism appears the most promising. In terms of the time-dependent many-body state $|\Psi(t)\rangle$ the current density is given by

$$
\mathbf{j}(\mathbf{r}, t)=\left\langle\Psi(t)\left|\hat{\mathbf{j}}_{\mathrm{p}}(\mathbf{r})\right| \Psi(t)\right\rangle+\langle\Psi(t)|\hat{n}(\mathbf{r})| \Psi(t)\rangle \mathbf{A}(\mathbf{r} t),
$$

where $\mathbf{A}$ is the applied vector potential, $\hat{n}(\mathbf{r})$ is the density operator and

$$
\hat{\mathbf{j}}_{\mathrm{p}}(\mathbf{r}, t)=\frac{1}{2 i} \sum_{\sigma}\left[\hat{\psi}^{\dagger}(\mathbf{x}) \nabla \hat{\psi}(\mathbf{x})-\nabla \hat{\psi}^{\dagger}(\mathbf{x}) \hat{\psi}(\mathbf{x})\right]
$$

is the paramagnetic current operator expressed in terms of the field operators $\hat{\psi}$ and $\hat{\psi}^{\dagger}$, where $\mathbf{x}=(\mathbf{r}, \sigma)$ is a space-spin index. In TDCDFT, this current density is calculated instead from a Kohn-Sham state $\left|\Psi_{s}(t)\right\rangle$ with a time-evolution determined by a non-interacting Kohn-Sham Hamiltonian $\hat{H}_{s}(t)$. This Hamiltonian contains an external Kohn-Sham vector field $\mathbf{A}_{s}$ (in a gauge where we absorb the scalar potentials in a vector potential) which is a functional of the current density. In this way, the photo-emission experiment can be modelled theoretically by time-propagation of Kohn-Sham orbitals after a suitable approximation for the Kohn-Sham vector potential $\mathbf{A}_{s}$ has been chosen. Indeed, some first calculations of this kind have been carried out recently. ${ }^{19}$

There are, however, two issues that remain unresolved. The first issue is the question whether TDCDFT allows for the determination of the kinetic energy distribution of the photo-electrons. The second issue is what the quality of the corresponding exchange-correlation kernels must be in order to account for many-body features such as plasmon losses. 
These are the two issues that we will address in this paper. The paper is divided as follows. In Sec. II, we briefly review the many-body approach to photo-emission where we stress the equations that are relevant for the connections to densityfunctional theory. In Sec. III, we give a description of TDCDFT in the language of Keldysh theory in order to make connection with the standard many-body approaches. We further give a discussion of the calculation of the kinetic energy distribution and the related very long range nonlocalities that are required in TDCDFT to calculate this property exactly. Finally, in Sec. IV we give our outlook and conclusions.

\section{MANY-BODY THEORY OF PHOTO-EMISSION}

\section{A. The photocurrent}

Here, we will present a short overview of the many-body approach to photo-emission in which we highlight the aspects relevant to the density functional treatment. We will follow the approach outlined by Almbladh. ${ }^{4}$ We assume that the many-body system is described by a Hamiltonian of the form

$$
\hat{H}(t)=\hat{H}_{0}+\hat{\Delta}(t),
$$

where $\hat{\Delta}$ describes the electromagnetic field applied for times $t>t_{0}$ and $\hat{H}_{0}$ is the many-body Hamiltonian of the sample before the field is applied. The time-evolution of the many-body state is described by the time-dependent Schrödinger equation

$$
i \partial_{t}|\Psi(t)\rangle=\hat{H}(t)|\Psi(t)\rangle
$$

with initial condition $\left|\Psi\left(t_{0}\right)\right\rangle=\left|\Phi_{0}\right\rangle$. We take $\left|\Phi_{0}\right\rangle$ to be the ground state of $\hat{H}_{0}$, i.e., $\hat{H}_{0}\left|\Phi_{0}\right\rangle=E_{0}\left|\Phi_{0}\right\rangle$ where $E_{0}$ is the ground state energy. If we know the state $|\Psi(t)\rangle$ then we can calculate all observables of interest. In the case of photo-emission the observable of interest is the current density outside the sample which describes the emission of photo-electrons. This amounts to the calculation of a onebody observable. In non-equilibrium many-body theory ${ }^{20}$ such observables can be calculated directly from the lesser Green's function defined as

$$
G^{<}\left(\mathbf{x} t, \mathbf{x}^{\prime} t^{\prime}\right)=i\left\langle\Phi_{0}\left|\hat{\psi}_{H}^{\dagger}\left(\mathbf{x}^{\prime} t^{\prime}\right) \hat{\psi}_{H}(\mathbf{x} t)\right| \Phi_{0}\right\rangle,
$$

where $\hat{A}_{H}(t)=\hat{U}\left(t_{0}, t\right) \hat{A} \hat{U}\left(t, t_{0}\right)$ is the Heisenberg form of the operator $\hat{A}$ with respect to the full Hamiltonian and $\hat{U}$ is the evolution operator of the system which in general is a time-ordered exponential. The current can then be calculated from

$$
\mathbf{j}_{\mathrm{p}}(\mathbf{r}, t)=-\left.\frac{1}{2} \sum_{\sigma}\left(\nabla-\nabla^{\prime}\right) G^{<}\left(\mathbf{x} t, \mathbf{x}^{\prime} t^{\prime}\right)\right|_{\mathbf{x}=\mathbf{x}^{\prime}}
$$

Here, we concentrate on the paramagnetic part of the current as we will see that the diamagnetic part only contributes to higher order in the applied field. Let us see what we get if we expand in powers of the electromagnetic coupling $\hat{\Delta}$. To do this we first expand the time-dependent many-body state in powers of $\hat{\Delta}$,

$$
|\Psi(t)\rangle=\sum_{n=0}^{\infty}\left|\Psi^{(n)}(t)\right\rangle
$$

where $\left|\Psi^{(n)}\right\rangle$ is the $n$th order term. In particular, $\left|\Psi^{(0)}(t)\right\rangle$ $=e^{-i E_{0}\left(t-t_{0}\right)}\left|\Phi_{0}\right\rangle$. The $k$ th order term in the expectation value for the current is then given by

$$
\mathbf{j}^{(k)}(\mathbf{r}, t)=\sum_{n+m=k}\left\langle\Psi^{(n)}(t)\left|\hat{\mathbf{j}}_{\mathrm{p}}(\mathbf{r})\right| \Psi^{(m)}(t)\right\rangle .
$$

If we are interested in the photo-emission current outside the sample then any term with $m=0$ or $n=0$ does not contribute since $\left|\Psi^{(0)}(t)\right\rangle$ is localized to the sample in position space and vanishes exponentially outside the sample. The diamagnetic current $n(\mathbf{r} t) \mathbf{A}(\mathbf{r} t)$ is even smaller since the lowest order contribution not involving $\left|\Psi^{(0)}(t)\right\rangle$ is third order in the applied field. The lowest order non-zero contribution to the photocurrent is therefore given by

$$
\mathbf{j}^{(2)}(\mathbf{r}, t)=\left\langle\Psi^{(1)}(t)\left|\hat{\mathbf{j}}_{\mathrm{p}}(\mathbf{r})\right| \Psi^{(1)}(t)\right\rangle .
$$

The other two lowest order terms $\left\langle\Psi^{(2)}(t)\left|\hat{\mathbf{j}}_{\mathrm{p}}(\mathbf{r})\right| \Psi^{(0)}(t)\right\rangle$ and $\left\langle\Psi^{(0)}(t)\left|\hat{\mathbf{j}}_{\mathrm{p}}(\mathbf{r})\right| \Psi^{(2)}(t)\right\rangle$ contributing to $\mathbf{j}^{(2)}$ are zero since we are assuming the photocurrent to be measured far outside the sample.

The calculation of $\mathbf{j}^{(2)}$ requires the knowledge of the first order change in the many-body state upon application of the field. This is readily calculated to be

$$
\left|\Psi^{(1)}(t)\right\rangle=-i \int_{t_{0}}^{t} d t^{\prime} \hat{U}_{0}\left(t, t^{\prime}\right) \hat{\Delta}\left(t^{\prime}\right) \hat{U}_{0}\left(t^{\prime}, t_{0}\right)\left|\Phi_{0}\right\rangle,
$$

where $\hat{U}_{0}\left(t, t^{\prime}\right)=e^{-i \hat{H}_{0}\left(t-t^{\prime}\right)}$ is the time-evolution operator of the unperturbed system. Using this expression we can write the photocurrent as

$$
\mathbf{j}^{(2)}(\mathbf{r}, t)=\int_{t_{0}}^{t} d t_{1} d t_{2}\left\langle\Phi_{0}\left|\hat{\Delta}_{H_{0}}\left(t_{2}\right) \hat{\mathbf{j}}_{\mathrm{p}, H_{0}}(\mathbf{r} t) \hat{\Delta}_{H_{0}}\left(t_{1}\right)\right| \Phi_{0}\right\rangle,
$$

where the operators are now in the Heisenberg representation with respect to $\hat{H}_{0}$. This is the starting expression for all our considerations. In many-body perturbation theory this expression is expanded in powers of the many-body interactions and can be represented as a diagrammatic series. To do this it will be convenient to define the equal-time lesser Green's function (or equivalently the one-particle density matrix) to second order in the external perturbation as

$$
\begin{aligned}
& G^{(2)<}\left(\mathbf{x} t, \mathbf{x}^{\prime} t\right) \\
& \quad=i \int_{t_{0}}^{t} d t_{1} d t_{2}\left\langle\Phi_{0}\left|\hat{\Delta}_{H_{0}}\left(t_{2}\right) \hat{\psi}_{H_{0}}^{\dagger}\left(\mathbf{x}^{\prime} t\right) \hat{\psi}_{H_{0}}(\mathbf{x} t) \hat{\Delta}_{H_{0}}\left(t_{1}\right)\right| \Phi_{0}\right\rangle
\end{aligned}
$$

as the Green's function has a well-known expansion in Feynman diagrams.

\section{B. Diagrammatic expansion}

To expand Eq. (6) into diagrams it will be convenient to write it as follows:

$$
\begin{aligned}
& G^{(2)<}\left(\mathbf{x} t, \mathbf{x}^{\prime} t\right) \\
& =i \int_{t_{0}}^{t} d t_{1} d t_{2}\left\langle\Phi_{0}\left|\hat{\Delta}_{+}\left(t_{2}\right) \hat{\psi}^{\dagger}\left(\mathbf{x}^{\prime}\right) \hat{\psi}(\mathbf{x}) \hat{\Delta}_{-}\left(t_{1}\right)\right| \Phi_{0}\right\rangle,
\end{aligned}
$$


where we defined

$$
\begin{aligned}
& \hat{\Delta}_{-}\left(t_{1}\right)=\hat{U}_{0}\left(t, t_{1}\right) \hat{\Delta}\left(t_{1}\right) \hat{U}_{0}\left(t_{1}, t_{0}\right), \\
& \hat{\Delta}_{+}\left(t_{2}\right)=\hat{U}_{0}\left(t_{0}, t_{2}\right) \hat{\Delta}\left(t_{2}\right) \hat{U}_{0}\left(t_{2}, t\right) .
\end{aligned}
$$

The operator $\hat{\Delta}_{-}\left(t_{1}\right)$ can now be expanded in time-ordered powers of the many-body interaction, whereas $\hat{\Delta}_{+}\left(t_{2}\right)$ can be expanded in anti-time-ordered powers of the interaction. Since it will not be our goal to give an overview of many-body theory, we restrict ourselves to the minimum which is required for understanding the connections to density functional theory. Within the language of Keldysh many-body theory ${ }^{20}$ we can say that the operator $\hat{\Delta}_{-}$is situated on the forward branch of the Keldysh contour whereas $\hat{\Delta}_{+}$is situated on the backward branch. This leads to an expansion of $G^{(2)}<$ in terms of the non-interacting Greens' functions,

$$
\begin{aligned}
& G_{--}\left(\mathbf{x} t, \mathbf{x}^{\prime} t^{\prime}\right)=-i\left\langle\chi_{0}\left|T\left[\hat{\psi}_{H}(\mathbf{x} t) \hat{\psi}_{H}^{\dagger}\left(\mathbf{x}^{\prime} t^{\prime}\right)\right]\right| \chi_{0}\right\rangle, \\
& G_{++}\left(\mathbf{x} t, \mathbf{x}^{\prime} t^{\prime}\right)=-i\left\langle\chi_{0}\left|\tilde{T}\left[\hat{\psi}_{H}(\mathbf{x} t) \hat{\psi}_{H}^{\dagger}\left(\mathbf{x}^{\prime} t^{\prime}\right)\right]\right| \chi_{0}\right\rangle, \\
& G_{-+}\left(\mathbf{x} t, \mathbf{x}^{\prime} t^{\prime}\right)=i\left\langle\chi_{0}\left|\hat{\psi}_{H}^{\dagger}\left(\mathbf{x}^{\prime} t^{\prime}\right) \hat{\psi}_{H}(\mathbf{x} t)\right| \chi_{0}\right\rangle, \\
& G_{+-}\left(\mathbf{x} t, \mathbf{x}^{\prime} t^{\prime}\right)=-i\left\langle\chi_{0}\left|\hat{\psi}_{H}(\mathbf{x} t) \hat{\psi}_{H}^{\dagger}\left(\mathbf{x}^{\prime} t^{\prime}\right)\right| \chi_{0}\right\rangle,
\end{aligned}
$$

where $T$ is the time-ordering operator, $\tilde{T}$ is the anti-timeordering operator, and $\left|\chi_{0}\right\rangle$ is the ground state of the noninteracting system and the operators are in the Heisenberg picture with respect to the noninteracting system. The Green's functions $G_{-+}$and $G_{+-}$are equivalently denoted as $G^{<}$and $G^{>}$. The vertices in the diagrams are labeled by - or + depending on whether they lie on the forward (-) or backward $(+)$ branch of the Keldysh contour. The bare Coulomb interactions will be denoted by wiggly lines and since these interactions are instantaneous they will always connect times on the same branch of the contour. Often the Green's function lines in the diagrams are dressed by self-energy insertions such that we can expand in skeleton diagrams (i.e., diagrams with self-energy insertions removed) but with dressed Green's function lines. Similarly, the interactions are often dressed to become screened interactions $W$ which now can connect vertices on different branches of the contour. Since the Green's function $G^{(2)<}$ has the same time on the ingoing and outgoing vertex the Green's function lines are commonly drawn closed back upon themselves to form triangles. In Fig. 1, we show the skeleton diagram expansion of $G^{(2)}<$ to lowest order in the screened interaction $W$ and the dressed Green's function $G$. Diagrams (a)-(c) are so-called no-loss diagrams whereas diagrams (d)-(f) describe energy losses of the photo-electron while leaving the sample. Diagrams (g) and (h) describe the renormalization of the photon-field inside the sample. For a more in-depth discussion we refer to Refs. 4 and 6.

\section{Spectral representation of the photocurrent}

Let us study the lowest order diagram in $W$ of Fig. 1. The structure of this diagram will also be relevant for the density functional case. It has the explicit form

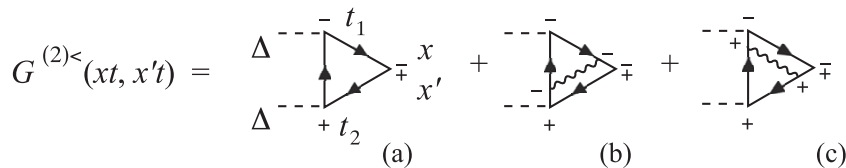

(c)

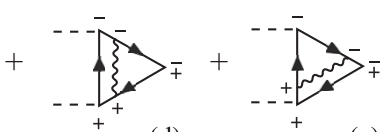

(d)

(e)
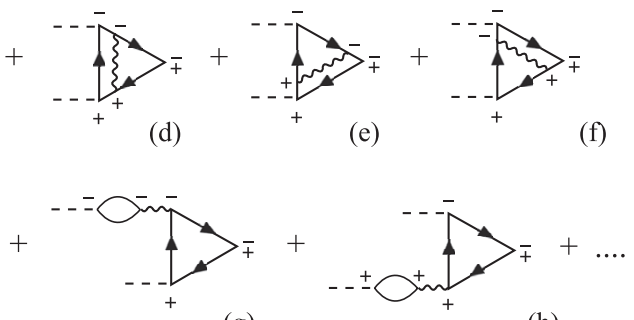

(g)

(h)

FIG. 1. Skeleton expansion of $G^{(2)}<$ in $G$ and $W$ to the first order in $W$.

$$
\begin{aligned}
G^{(2)<} & \left(\mathbf{x} t, \mathbf{x}^{\prime} t\right) \\
= & -\int_{-\infty}^{t} d t_{1} d t_{2} \\
& \quad \times\left\langle\mathbf{x}\left|\hat{\mathcal{G}}^{--}\left(t, t_{1}\right) \hat{\Delta}\left(t_{1}\right) \hat{\mathcal{G}}^{-+}\left(t_{1}, t_{2}\right) \hat{\Delta}\left(t_{2}\right) \hat{\mathcal{G}}^{++}\left(t_{2}, t\right)\right| \mathbf{x}^{\prime}\right\rangle,
\end{aligned}
$$

where the minus sign originates from integration on the \pm branch and we used the convenient notation

$$
G_{\alpha \alpha^{\prime}}\left(\mathbf{x} t, \mathbf{x}^{\prime} t^{\prime}\right)=\left\langle\mathbf{x}\left|\hat{\mathcal{G}}^{\alpha \alpha^{\prime}}\left(t, t^{\prime}\right)\right| \mathbf{x}^{\prime}\right\rangle .
$$

Now the lesser Green's function $\left\langle\mathbf{x}\left|\hat{\mathcal{G}}^{<}\right| \mathbf{y}\right\rangle$ vanishes for spatial coordinates outside the sample as it depends only on the occupied states of the unperturbed system. We can therefore write in our case that

$$
\begin{aligned}
& \hat{\mathcal{G}}^{--}\left(t, t^{\prime}\right)=\theta\left(t-t^{\prime}\right) \hat{\mathcal{G}}^{>}\left(t, t^{\prime}\right)=\hat{\mathcal{G}}^{R}\left(t, t^{\prime}\right), \\
& \hat{\mathcal{G}}^{++}\left(t, t^{\prime}\right)=\theta\left(t^{\prime}-t\right) \hat{\mathcal{G}}^{>}\left(t, t^{\prime}\right)=-\hat{\mathcal{G}}^{A}\left(t, t^{\prime}\right),
\end{aligned}
$$

where the retarded and advanced propagators are defined as

$$
\begin{aligned}
& \hat{\mathcal{G}}^{R}\left(t, t^{\prime}\right)=\theta\left(t-t^{\prime}\right)\left[\hat{\mathcal{G}}^{>}-\hat{\mathcal{G}}^{<}\right]\left(t, t^{\prime}\right), \\
& \hat{\mathcal{G}}^{A}\left(t, t^{\prime}\right)=-\theta\left(t^{\prime}-t\right)\left[\hat{\mathcal{G}}^{>}-\hat{\mathcal{G}}^{<}\right]\left(t, t^{\prime}\right) .
\end{aligned}
$$

In terms of these propagators the expression (7) attains the form

$$
\begin{aligned}
G^{(2)} & <\left(\mathbf{x} t, \mathbf{x}^{\prime} t\right) \\
& =\int_{-\infty}^{t} d t_{1} d t_{2} \\
& \times\left\langle\mathbf{x}\left|\hat{\mathcal{G}}^{R}\left(t, t_{1}\right) \hat{\Delta}\left(t_{1}\right) \hat{\mathcal{G}}^{<}\left(t_{1}, t_{2}\right) \hat{\Delta}\left(t_{2}\right) \hat{\mathcal{G}}^{A}\left(t_{2}, t\right)\right| \mathbf{x}^{\prime}\right\rangle
\end{aligned}
$$

This expression is valid for general time-dependent perturbations. Let us, however, restrict ourselves to a mono-chromatic perturbation for $t>t_{0}$ of the form

$$
\hat{\Delta}(t)=\hat{w} e^{-i \Omega t}+\hat{w}^{\dagger} e^{i \Omega t}=\sum_{\rho= \pm} \hat{w}_{\rho} e^{i \rho \Omega t},
$$

where $\Omega>0$ and where we define $\hat{w}_{-}=\hat{w}$ and $\hat{w}_{+}=\hat{w}^{\dagger}$. Inserting this expression into Eq. (8) and assuming that $t_{0}$ is very far into the past, we then obtain that 


$$
\begin{aligned}
G^{(2)<} & \left(\mathbf{x} t, \mathbf{x}^{\prime} t\right) \\
= & \sum_{\rho, \eta= \pm} e^{-i(\eta+\rho) \Omega t} \\
& \times \int \frac{d \omega}{2 \pi}\left\langle\mathbf{x}\left|\hat{\mathcal{G}}^{R}(\omega+\eta \Omega) \hat{w}_{\eta} \hat{\mathcal{G}}^{<}(\omega) \hat{w}_{\rho} \hat{\mathcal{G}}^{A}(\omega-\rho \Omega)\right| \mathbf{x}^{\prime}\right\rangle,
\end{aligned}
$$

where we wrote the Green's functions as Fourier transforms

$$
\hat{\mathcal{G}}\left(t, t^{\prime}\right)=\int \frac{d \omega}{2 \pi} e^{-i \omega\left(t-t^{\prime}\right)} \hat{\mathcal{G}}(\omega) .
$$

We can now manipulate this expression in an expansion in terms of the free particle Green's functions $\hat{\mathcal{G}}_{0}^{R, A}$ of the photoelectron leaving the sample. After some manipulations which are presented in the Appendix we find that outside the sample the lesser Green's function attains the form

$$
\begin{aligned}
G^{(2)<}\left(\mathbf{x} t, \mathbf{x}^{\prime} t\right)= & \frac{\delta_{\sigma \sigma^{\prime}}}{4 \pi^{2}|\mathbf{r}|\left|\mathbf{r}^{\prime}\right|} \\
& \times \int \frac{d \omega}{2 \pi} e^{i q\left(|\mathbf{r}|-\left|\mathbf{r}^{\prime}\right|\right)}\left\langle\varphi_{q \hat{\mathbf{r}}}\left|\hat{w}^{\dagger} \hat{\mathcal{G}}^{<}(\omega) \hat{w}\right| \varphi_{q \hat{\mathbf{r}}^{\prime}}\right\rangle,
\end{aligned}
$$

where $q^{2} / 2=\omega+\Omega$ is the kinetic energy of the photoelectron and $\hat{\mathbf{r}}=\mathbf{r} /|\mathbf{r}|$ is the unit vector pointing from the sample to the detector. If we further define $\mathbf{q}=q \hat{\mathbf{r}}$ then the state $\left|\varphi_{\mathbf{q}}\right\rangle$ is a quasi-particle state satisfying the equation

$$
\left[\hat{h}+\hat{\Sigma}^{A}(\omega+\Omega)\right]\left|\varphi_{\mathbf{q}}\right\rangle=(\omega+\Omega)\left|\varphi_{\mathbf{q}}\right\rangle,
$$

where $\hat{h}$ is the one-body part of $\hat{H}_{0}$ and $\hat{\Sigma}^{A}$ is the advanced self-energy. We can now use Eq. (2) to calculate the current density which gives

$$
\mathbf{j}^{(2)}(\mathbf{r} t)=\frac{\hat{\mathbf{r}}}{4 \pi^{2}|\mathbf{r}|^{2}} F(\hat{\mathbf{r}}),
$$

where

$$
F(\hat{\mathbf{r}})=\int_{-\infty}^{\mu} \frac{d \omega}{2 \pi} q\left\langle\varphi_{\mathbf{q}}\left|\hat{w}^{\dagger} \hat{\mathcal{A}}(\omega) \hat{w}\right| \varphi_{\mathbf{q}}\right\rangle
$$

where we neglected terms of order $1 /|\mathbf{r}|^{3}$, and used the fluctuation-dissipation relation $\hat{\mathcal{G}}^{<}(\omega)=$ if $(\omega-\mu) \hat{\mathcal{A}}(\omega)$ with $f$ the Fermi function at chemical potential $\mu$ and $\hat{\mathcal{A}}(\omega)$ $=i\left[\hat{\mathcal{G}}^{R}(\omega)-\hat{\mathcal{G}}^{A}(\omega)\right]$ the spectral function.

In the experiment, one measures the flux of the current through a space angle $d \bar{\Omega}$,

$$
J_{d \bar{\Omega}}=\int_{d \bar{\Omega}} \mathbf{j} \cdot d \mathbf{S}
$$

through a spherical surface $\mathbf{S}$ of radius $|\mathbf{r}|$. If we further define $\epsilon=q^{2} / 2=\omega+\Omega$ to be the kinetic energy of the photoelectron as a new variable, then we can write for the current per space angle

$$
\frac{\partial J}{\partial \bar{\Omega}}(\hat{\mathbf{r}})=\frac{1}{4 \pi^{2}} \int_{-\infty}^{\mu+\Omega} \frac{d \epsilon}{2 \pi} \sqrt{2 \epsilon}\left\langle\varphi_{\mathbf{q}}\left|\hat{w}^{\dagger} \hat{\mathcal{A}}(\epsilon-\Omega) \hat{w}\right| \varphi_{\mathbf{q}}\right\rangle .
$$

Now, in an experiment also the kinetic energy of the photoelectron can be measured. In this way, the photocurrent can be split into energy contributions and we can then write

$$
\frac{\partial^{2} J}{\partial \bar{\Omega} \partial \epsilon}(\hat{\mathbf{r}})=\frac{\sqrt{2 \epsilon}}{(2 \pi)^{3}}\left\langle\varphi_{\mathbf{q}}\left|\hat{w}^{\dagger} \hat{\mathcal{A}}(\epsilon-\Omega) \hat{w}\right| \varphi_{\mathbf{q}}\right\rangle .
$$

By measuring both the direction and energy of the photoelectron the right hand side of this expression can be measured.

\section{DENSITY FUNCTIONAL THEORY FOR PHOTO-EMISSION}

\section{A. Current density functional theory}

In this section, we give a short overview of the basic equations of TDCDFT and its connection to many-body perturbation theory. A much more detailed exposition can be found in Refs. 21 and 22. So far we did not specify the precise form of the perturbation. In general its form will be given by that of an electromagnetic field described by a time-dependent scalar potential and a vector potential A. However, we can always choose a gauge where the time-dependent fields are absorbed in a vector potential. Static potentials, such as the potentials due to atomic nuclei, may still be described in terms of a scalar potential absorbed in $\hat{H}_{0}$. If we do this we can write the perturbation as

$$
\hat{\Delta}(t)=\int d \mathbf{r} \hat{\mathbf{j}}_{\mathrm{p}}(\mathbf{r}) \cdot \mathbf{A}(\mathbf{r}, t)+\frac{1}{2} \int d \mathbf{r} \hat{n}(\mathbf{r}) \mathbf{A}^{2}(\mathbf{r}, t) .
$$

We can then define a functional $\tilde{F}[\mathbf{A}]$ of the vector potential by

$$
\tilde{F}[\mathbf{A}]=i \ln \left\langle\Phi_{0}\left|T_{\gamma} e^{-i \int_{\gamma} d z \hat{H}(z)}\right| \Phi_{0}\right\rangle,
$$

where $T_{\gamma}$ denotes contour ordering on the Keldysh contour $\gamma$ with contour time $z \cdot{ }^{20}$ This functional has the derivative

$$
\frac{\delta \tilde{F}}{\delta \mathbf{A}(\mathbf{r}, z)}=\mathbf{j}_{\mathrm{p}}(\mathbf{r}, z)+n(\mathbf{r}, z) \mathbf{A}(\mathbf{r}, z)=\mathbf{j}(\mathbf{r}, z),
$$

where $\mathbf{j}_{\mathrm{p}}$ is the paramagnetic current and $\mathbf{j}$ is the physical gauge-invariant current. This physical current is the central object of time-dependent current-density-functional theory. ${ }^{11,17}$ We can make a current functional $F[\mathbf{j}]$ by a Legendre transform

$$
F[\mathbf{j}]=-\tilde{F}[\mathbf{A}]+\int_{\gamma} d \mathbf{r} d z \mathbf{j}(\mathbf{r}, z) \cdot \mathbf{A}(\mathbf{r}, z),
$$

which has the property

$$
\frac{\delta F}{\delta \mathbf{j}(\mathbf{r}, z)}=\mathbf{A}(\mathbf{r}, z) .
$$

The whole derivation did not depend on the specific form of the many-body interactions in $\hat{H}_{0}$. The only thing that we assumed was that there is a one-to-one relation between the physical current and the vector potential in our specific gauge given the initial state $\left|\Phi_{0}\right\rangle .{ }^{17,18}$ We could therefore have done the same derivation for a non-interacting system with initial state $\left|\Phi_{0, s}\right\rangle$ and obtain a current functional which we call $F_{s}[\mathbf{j}]$. We now assume that the functionals $F[\mathbf{j}]$ and $F_{s}[\mathbf{j}]$ have the same domain. We then define the exchange-correlation (xc) current functional $F_{\mathrm{xc}}$ as

$$
F_{\mathrm{xc}}[\mathbf{j}]=F_{s}[\mathbf{j}]-F[\mathbf{j}]-F_{\mathrm{H}}[\mathbf{j}],
$$


where

$$
F_{\mathrm{H}}[\mathbf{j}]=\frac{1}{2} \int d \mathbf{r} d \mathbf{r}^{\prime} \int_{\gamma} d z n(\mathbf{r}, z) n\left(\mathbf{r}^{\prime}, z\right) v\left(\mathbf{r}-\mathbf{r}^{\prime}\right),
$$

where $v$ is the two-body interaction and where the density $n(\mathbf{r}, z)$ is regarded a functional of the current through the continuity equation. Differentiation of Eq. (16) with respect to $\mathbf{j}$ gives

$$
\mathbf{A}_{\mathrm{xc}}=\mathbf{A}_{s}-\mathbf{A}-\mathbf{A}_{\mathrm{H}},
$$

where we defined $\mathbf{A}_{\mathrm{xc}}=\delta F_{\mathrm{xc}} / \delta \mathbf{j}$ and $\mathbf{A}_{\mathrm{H}}=\delta F_{\mathrm{H}} / \delta \mathbf{j}$. The potential $\mathbf{A}_{s}$ is the vector potential that for a non-interacting system gives the current density $\mathbf{j}$. This system is called the Kohn-Sham system and $\mathbf{A}_{s}$ will be called the Kohn-Sham vector potential. If we take the initial state $\left|\Phi_{s, 0}\right\rangle$ to be a Kohn-Sham ground state then the current can be calculated by solving the Kohn-Sham single-particle equations,

$$
\left[\frac{1}{2}\left(-i \nabla+\mathbf{A}_{s}(\mathbf{r}, t)\right)^{2}+v_{\mathrm{ext}}(\mathbf{r})\right] \phi_{j}(\mathbf{r}, t)=i \partial_{t} \phi_{j}(\mathbf{r}, t),
$$

where $v_{\text {ext }}$ is the static external field of the unperturbed system. If we differentiate Eq. (17) with respect to $\mathbf{j}$ we obtain

$$
\frac{\delta A_{\mathrm{Hxc}, \mu}(\mathbf{r}, z)}{\delta j_{v}\left(\mathbf{r}^{\prime}, z^{\prime}\right)}=\frac{\delta A_{s, \mu}(\mathbf{r}, z)}{\delta j_{v}\left(\mathbf{r}^{\prime}, z^{\prime}\right)}-\frac{\delta A_{\mu}(\mathbf{r}, z)}{\delta j_{v}\left(\mathbf{r}^{\prime}, z^{\prime}\right)},
$$

where $\mathbf{A}_{\mathrm{Hxc}}$ is the sum of the Hartree and xc vector potentials. The indices $\mu$ and $v$ label the three components of the vectors. The quantity on the left hand side is usually called the Hartree and xc kernel $f_{\mathrm{Hxc}}$ which can be split naturally into a Hartree part $f_{\mathrm{H}}$ and an xc part $f_{\mathrm{xc}}$. The derivatives $\delta A_{\mu} / \delta j_{v}$ represent the inverse of the current-current response function given by

$$
\begin{aligned}
\chi_{\mu \nu}\left(\mathbf{r} z, \mathbf{r}^{\prime} z^{\prime}\right)= & \frac{\delta j_{\mu}(\mathbf{r}, z)}{\delta A_{\nu}\left(\mathbf{r}^{\prime}, z^{\prime}\right)}=\delta_{\mu \nu} n_{0}(\mathbf{r}) \delta\left(\mathbf{r}-\mathbf{r}^{\prime}\right) \delta\left(z, z^{\prime}\right) \\
& -i\left\langle\Phi_{0}\left|T_{\gamma}\left\{\Delta \hat{j}_{\mathrm{p}, \mu, H}(\mathbf{r}, z) \Delta \hat{j}_{\mathrm{p}, \nu, H}\left(\mathbf{r}^{\prime} z^{\prime}\right)\right\}\right| \Phi_{0}\right\rangle,
\end{aligned}
$$

where the first part arises from the diamagnetic current and the second from the paramagnetic one and we further defined the current fluctuation operator by $\Delta \hat{j}_{\mathrm{p}, \mu, H}=\hat{j}_{\mathrm{p}, \mu, H}$ $-\left\langle\hat{j}_{\mathrm{p}, \mu, H}\right\rangle$. We have a similar response function $\chi_{s}=\delta j / \delta A_{s}$ for the Kohn-Sham system. From Eq. (18) we see then that we can write

$$
\chi=\chi_{s}+\chi_{s} \cdot f_{\mathrm{Hxc}} \cdot \chi,
$$

where the dot product indicates a matrix product with respect to the indices and integration over space-time variables on the contour. This is the central equation of density functional theory for linear response. ${ }^{11}$ Approximations for $f_{\mathrm{Hxc}}$ can be found by expanding $F_{\mathrm{xc}}$ in diagrams. Some explicit examples of this will be given below.

\section{B. Photo-emission in current-density functional theory}

The photocurrent within TDCDFT can be calculated as

$$
\mathbf{j}_{s}^{(k)}(\mathbf{r}, t)=\sum_{n+m=k}\left\langle\Psi_{s}^{(n)}(t)\left|\hat{\mathbf{j}}_{\mathrm{p}}(\mathbf{r})\right| \Psi_{s}^{(m)}(t)\right\rangle
$$

where were have expanded the Kohn-Sham state in powers of the variation of the Kohn-Sham field [cf. Eq. (3)]. The

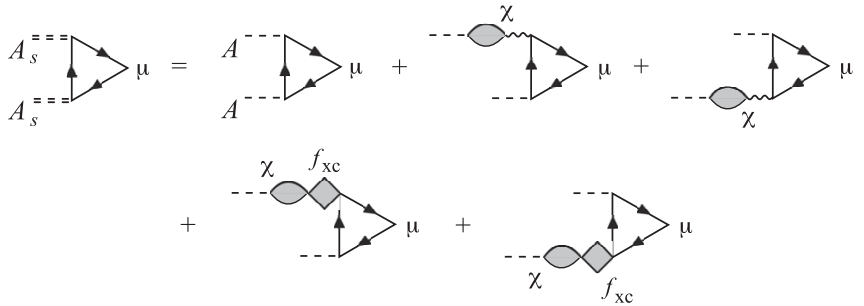

FIG. 2. Diagrammatic expansion for the photocurrent in TDCDFT to linear order in $f_{\mathrm{Hxc}}$ and $\chi$ (see also Fig. 1). The external vertex has a label $\mu$ corresponding to the action of a current operator and the Hartree kernel $f_{\mathrm{H}}$ is indicated by a wiggly line.

current density of TDCDFT is exactly the same as the current density of the real system and therefore $\mathbf{j}(\mathbf{r}, t)=\mathbf{j}_{s}(\mathbf{r}, t)$. Since we are measuring the photocurrent far outside the sample and the initial Kohn-Sham state is a Slater determinant of Kohn-Sham orbitals that vanish exponentially outside the sample the terms with $m=0$ and/or $n=0$ do not contribute. Therefore, as in Sec. II A where we expanded in powers of the physical vector potential, the lowest order contribution in the Kohn-Sham field to the photocurrent is

$$
\mathbf{j}_{s}^{(2)}(\mathbf{r}, t)=\left\langle\Psi_{s}^{(1)}(t)\left|\hat{\mathbf{j}}_{\mathrm{p}}(\mathbf{r})\right| \Psi_{s}^{(1)}(t)\right\rangle .
$$

Since the Kohn-Sham field $\mathbf{A}_{s}[\mathbf{A}]$ is to lowest order linear in $\mathbf{A}$ we have that $\mathbf{j}_{s}^{(2)}(\mathbf{r}, t)=\mathbf{j}^{(2)}(\mathbf{r}, t)+O\left(\mathbf{A}^{3}\right)$. The difference with Eq. (4) is that the initial state $\left|\Phi_{0}\right\rangle$ is the Kohn-Sham initial state $\left|\Phi_{0, s}\right\rangle$ and that the perturbation $\hat{\Delta}$ is replaced by a Kohn-Sham perturbation $\hat{\Delta}_{s}$. Since there are no many-body interactions in the Kohn-Sham system the diagrammatic form of the current is simply given by the left hand side diagram in Fig. 2.

To write this diagram in terms of the applied field $\mathbf{A}$ we need to expand the Kohn-Sham field $\mathbf{A}_{s}$ in terms of $\mathbf{A}$. To lowest order this gives

$$
A_{s, \mu}(1)=\sum_{\nu} \int_{\gamma} d 2 K_{\mu \nu}(1,2) A_{\nu}(2)
$$

where

$$
\begin{aligned}
K_{\mu \nu}(1,2) & =\frac{\delta A_{\mu, s}(1)}{\delta A_{\nu}(2)} \\
& =\delta_{\mu \nu} \delta(1,2)+\sum_{\rho} \int_{\gamma} d 3 f_{\mathrm{Hxc}, \mu \rho}(1,3) \chi_{\rho \nu}(3,2)
\end{aligned}
$$

and we used the short notation $j=\left(\mathbf{r}_{j}, z_{j}\right)$. This expression can be written diagrammatically as in Fig. 3.

When we insert this diagrammatic representation in the diagrams for the current we obtain the graphical expansion on the right hand side of Fig. 2, in which we only displayed terms up to linear order in $f_{\mathrm{Hxc}}$ and $\chi$. We note that this gives

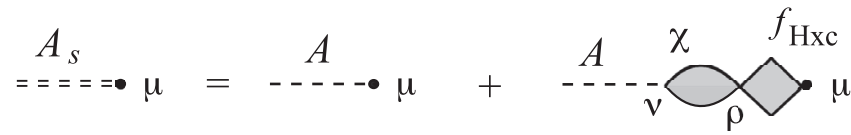

FIG. 3. Diagrammatic representation of the Kohn-Sham field $\mathbf{A}_{s}$ in terms of the applied filed $\mathbf{A}$ (see Eq. (20)). 
a rather different expansion than the one that we found for the expressions in many-body theory. In particular, we see that all the exchange-correlation contributions to the current amount to an effective renormalization of the photon field as there are no terms connecting the different legs of the triangle. Only the diagrams (g) and $(\mathrm{h})$ in Fig. 1 have a direct correspondence with the second and third diagram after the equal sign in Fig. 2 as both represent a renormalization due to the Hartree field.

Let us express the Kohn-Sham current in a frequency dependent form. If we take the external vector potential to be of the monochromatic form

$$
\mathbf{A}(\mathbf{r}, t)=\mathbf{a}(\mathbf{r}) e^{-i \Omega t}+\mathbf{a}^{*}(\mathbf{r}) e^{i \Omega t}
$$

then we can write

$$
\hat{\Delta}(t)=\hat{w} e^{-i \Omega t}+\hat{w}^{\dagger} e^{i \Omega t}
$$

where we neglected terms of order $\mathbf{A}^{2}$ and defined

$$
\hat{w}=\int d \mathbf{r} \hat{\mathbf{j}}_{\mathrm{p}}(\mathbf{r}) \cdot \mathbf{a}(\mathbf{r}) .
$$

Within linear response also the Kohn-Sham field has this form

$$
\mathbf{A}_{s}(\mathbf{r}, t)=\mathbf{a}_{s}(\mathbf{r}, \Omega) e^{-i \Omega t}+\mathbf{a}_{s}^{*}(\mathbf{r}, \Omega) e^{i \Omega t},
$$

where

$$
a_{\mu, s}(\mathbf{r}, \Omega)=\sum_{\nu} \int d \mathbf{r}^{\prime} K_{\mu \nu}^{R}\left(\mathbf{r}, \mathbf{r}^{\prime} ; \Omega\right) a_{\nu}\left(\mathbf{r}^{\prime}\right)
$$

and where $K_{\mu \nu}^{R}(\Omega)$ is the Fourier transform of the retarded component of $K_{\mu \nu}$ evaluated at the photon frequency $\Omega$. Then if we define

$$
\hat{w}_{s}=\int d \mathbf{r} \hat{\mathbf{j}}(\mathbf{r}) \cdot \mathbf{a}_{s}(\mathbf{r}, \Omega)
$$

we have that the function $F(\hat{\mathbf{r}})$ of (13) has the following expression in DFT

$$
F(\hat{\mathbf{r}})=\int_{-\infty}^{\mu} \frac{d \omega}{2 \pi} q\left\langle\varphi_{s, \mathbf{q}}\left|\hat{w}_{s}^{\dagger} \hat{\mathcal{A}}_{s}(\omega) \hat{w}_{s}\right| \varphi_{s, \mathbf{q}}\right\rangle .
$$

Here, $\hat{\mathcal{A}}_{s}(\omega)$ is the Kohn-Sham spectral function

$$
\hat{\mathcal{A}}_{s}(\omega)=2 \pi \sum_{j}\left|\phi_{j}\right\rangle\left\langle\phi_{j}\right| \delta\left(\omega-\epsilon_{j}\right),
$$

where $\epsilon_{j}$ and $\left|\phi_{j}\right\rangle$ are the Kohn-Sham one-particle energies and eigenstates and the photo-electron orbital $\left|\varphi_{s, \mathbf{q}}\right\rangle$ satisfies the equation

$$
\hat{h}_{s}\left|\varphi_{s, \mathbf{q}}\right\rangle=\frac{q^{2}}{2}\left|\varphi_{s, \mathbf{q}}\right\rangle
$$

with incoming plane wave boundary condition. Here, $\hat{h}_{s}$ is the one-body Kohn-Sham Hamiltonian of the unperturbed system. Since the highest occupied Kohn-Sham orbital energy is equal to minus the ionization energy, $\epsilon_{N}=-I$, (provided we choose a gauge where the potential is zero at infinity, see Ref. 23) we have $\mu=-I$ and therefore $\mu$ is the same for the true and the Kohn-Sham system. If we insert the expression for the spectral function operator into Eq. (22) we find that

$$
F(\hat{\mathbf{r}})=\sum_{\epsilon_{j} \leq \mu} q_{j}\left|\left\langle\phi_{j}\left|\hat{w}_{s}\right| \varphi_{s, q_{j} \hat{\mathbf{r}}}\right\rangle\right|^{2},
$$

where we defined $\epsilon_{j}+\Omega=q_{j}^{2} / 2$. This is an exact alternative expression for $F(\hat{\mathbf{r}})$ of Eq. (13). To expose its physical content we have to study explicit approximations to the xckernel $f_{\mathrm{xc}}$. This will be done in Sec. III C using diagrammatic expansions.

\section{Diagrammatic approximations for $f_{\mathrm{xc}}$}

We will give here a brief discussion of the diagrammatic expansion of $f_{\mathrm{xc}} \cdot{ }^{24-32}$ The starting point of the discussion is the equation

$$
\begin{aligned}
\frac{\delta F_{\mathrm{xc}}}{\delta A_{s, \mu}(1)} & =\sum_{v} \int_{\gamma} d 2 \frac{\delta F_{\mathrm{xc}}}{\delta j_{v}(2)} \frac{\delta j_{v}(2)}{\delta A_{s, \mu}(1)} \\
& =\sum_{v} \int_{\gamma} d 2 \chi_{s, \mu v}(1,2) A_{x c, v}(2),
\end{aligned}
$$

where we used the symmetry in $\mu$ and $v$ of $\chi_{s}$. We now assume that $F_{\mathrm{xc}}$ is given by an expansion in Kohn-Sham Green's functions $G_{s} .{ }^{33}$ Then the left hand side can be written as

$$
\frac{\delta F_{\mathrm{xc}}}{\delta A_{s, \mu}(1)}=\int_{\gamma} d 2 d 3 \frac{\delta F_{\mathrm{xc}}}{\delta G_{s}(2,3)} G_{s}(2,1) \bar{j}_{\mu}\left(\mathbf{r}_{1}\right) G_{s}(1,3),
$$

where we used ${ }^{21}$

$$
\frac{\delta G_{s}(2,3)}{\delta A_{s, \mu}(1)}=G_{s}(2,1) \bar{j}_{\mu}\left(\mathbf{r}_{1}\right) G_{s}(1,3)
$$

and defined

$$
\bar{j}_{\mu}(\mathbf{r})=\frac{1}{2 i}\left(\vec{\partial}_{\mu}-\overleftarrow{\partial}_{\mu}\right)
$$

If we call

$$
\frac{\delta F_{\mathrm{xc}}}{\delta G_{s}(2,3)}=\Sigma_{\mathrm{xc}}(3,2),
$$

then we can write Eq. (24) as

$$
\begin{aligned}
& \sum_{v} \int_{\gamma} d 2 \chi_{s, \mu v}(1,2) A_{\mathrm{xc}, v}(2) \\
& \quad=\int_{\gamma} d 2 d 3 \Sigma_{\mathrm{xc}}(3,2) G_{s}(2,1) \bar{j}_{\mu}\left(\mathbf{r}_{1}\right) G_{s}(1,3),
\end{aligned}
$$

which has the structure of a linearized Sham-Schlüter equation $^{29,34,35}$ as displayed in Fig. 4.

If we differentiate this equation once again with respect to $\mathbf{A}_{s}$ we obtain an integral equation for $f_{\mathrm{xc}}$ given by

$$
\begin{aligned}
& \sum_{\nu \lambda} \int_{\gamma} d 2 d 4 \chi_{s, \mu \nu}(1,2) f_{\mathrm{xc}, \nu \lambda}(2,4) \chi_{s, \lambda \kappa}(4,3) \\
& =Q_{\mu \kappa}(1,3)-\int_{\gamma} d 2 \chi_{s, \mu \nu \kappa}^{(2)}(1,2,3) A_{\mathrm{xc}, \nu}(2),
\end{aligned}
$$

FIG. 4. Diagrammatic representation of the integral equation for $\mathbf{A}_{\mathrm{xc}}$ (see Eq. (25)). 


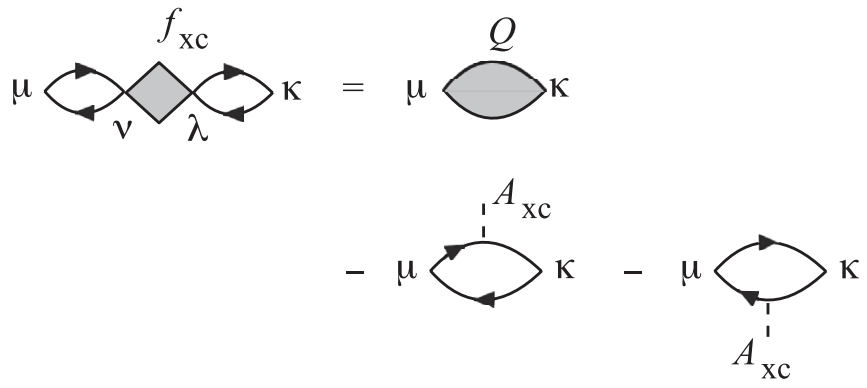

FIG. 5. Diagrammatic representation for the integral equation for $f_{\mathrm{xc}}$ (see Eq. (26)).

where we defined

$$
Q_{\mu \kappa}(1,3)=\frac{\delta^{2} F_{\mathrm{xc}}}{\delta A_{s, \mu}(1) \delta A_{s, \kappa}(3)}
$$

as well as the second order Kohn-Sham response function

$$
\chi_{s, \mu \nu \kappa}^{(2)}(1,2,3)=\frac{\delta \chi_{\mu \nu}(1,2)}{\delta A_{s, \kappa}(3)} .
$$

The corresponding integral equation for $f_{\mathrm{xc}}$ is displayed diagrammatically in Fig. 5. The diagrammatic structure of Eq. (26) has been studied in detail in Ref. 29 in which explicit diagrammatic expansions were derived from a LuttingerWard functional. For the case of the simple exchange approximation to $\Sigma_{\mathrm{xc}}$, for instance, we have

$$
\Sigma_{\mathrm{xc}}(1,2)=-i v(1,2) G_{s}(1,2),
$$

where $v(1,2)=\delta\left(z, z^{\prime}\right) v\left(\mathbf{r}_{1}-\mathbf{r}_{2}\right)$ is the bare many-body interaction. The corresponding diagrammatic expression for $Q_{\mu \kappa}$ is displayed in Fig. 6. A more advanced approximation will be discussed below. But before we do that we first discuss the diagrammatic expansion of the photocurrent within TDCDFT.

\section{Diagrams for the photocurrent}

We have seen that we can derive approximate expression for the xc-kernel of TDCDFT on the basis of many-body perturbation theory. A natural question to ask at this point would be how to relate these expressions to the Feynman diagrams for the photocurrent derived directly from many-body theory, such as the diagrams displayed in Fig. 1. The situation is complicated by the fact that we do not have a direct diagrammatic expression of $f_{\mu \nu, \mathrm{xc}}$ but rather one that is convoluted with two Kohn-Sham response functions as in Eq. (26). This is a consequence of the fact that we are working in the zero-temperature formalism where the memory of initial correlations and initial-state dependence is lost. This allows us to work with time-ordered quantities that depend on the time-difference only and, therefore, can be Fourier transformed. However, as it was first realized by Mearns and

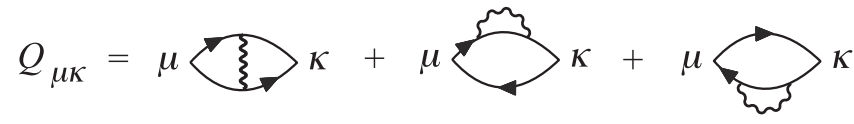

FIG. 6. Exchange-only approximation to $Q_{\mu \kappa}$.

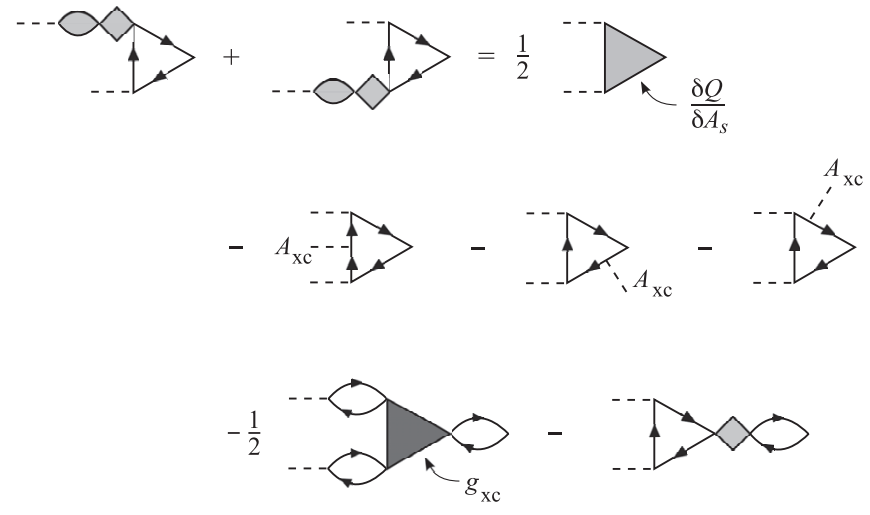

FIG. 7. Diagrammatic expansion of the photocurrent obtained from differentiating the integral equation of the xc-kernel and integrating with the external fields.

$\mathrm{Kohn}^{36}$ and recently discussed by Hellgren and von Barth, ${ }^{31}$ there are frequencies at which $\chi_{s}$ is not invertible, thus preventing a direct diagrammatic expansion of $f_{\mathrm{xc}}$ to be inserted into the diagrams of Fig. $2 .{ }^{37}$

In order to generate three-point diagrams, we can differentiate Fig. 5 another time with respect to $\mathbf{A}_{s}$. If we do this and collect our results we find an expression which we display graphically in Fig. 7, where after differentiation we integrated two of the external vertices with the external field $\hat{\Delta}(t)$. In this expression, we defined the higher-order xc-kernel $g_{\text {xc }}$ by

$$
g_{\mathrm{xc}, \mu \nu \tau}(1,2,3)=\frac{\delta f_{\mathrm{xc}, \mu \nu}(1,2)}{\delta j_{\tau}(3)} .
$$

The appearance of $g_{\mathrm{xc}}$ is not surprising given the fact that the photoemission problem is nonlinear in the external field. The first filled triangle on the right hand side of Fig. 7 represents half of the derivative $\delta Q / \delta A_{s}$. For the exchange-only approximation these diagrams (integrations with the external field) are shown in Fig. 8. The last two diagrams in Fig. 7 are exponentially small outside the sample as they contain the response function with a coordinate in the position of the detector.

Before continuing our analysis we observe that in the proximity of the sample the last two diagrams in Fig. 7 are not the only contributions to add to the photocurrent. In fact, the photocurrent has a first-order contribution as well

$$
\begin{aligned}
\mathbf{j}_{s}^{(1)}(1) & =\left\langle\Psi_{s}^{(0)}\left(t_{1}\right)\left|\hat{\mathbf{j}}_{\mathrm{p}}\left(\mathbf{r}_{1}\right)\right| \Psi_{s}^{(1)}\left(t_{1}\right)\right\rangle+\text { c.c. } \\
& =\int d 2 \chi_{s}(1,2) \delta \mathbf{A}_{s}(2),
\end{aligned}
$$

which can be discarded only for $\left|\mathbf{r}_{1}\right| \rightarrow \infty$. Let us expand this equation up to second order in the true external field $\mathbf{A}$. We

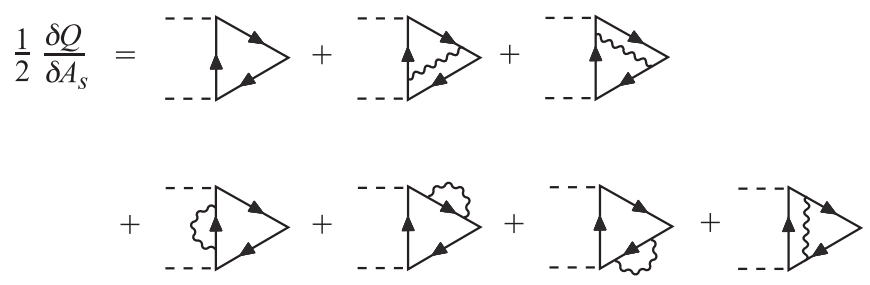

FIG. 8. The exchange-only graphs contribution to the photocurrent. 
have

$$
\begin{aligned}
\delta A_{s, \mu}(1)= & \sum_{\nu} \int \frac{\delta A_{s, \mu}(1)}{\delta A_{\tau}(2)} \delta A_{\tau}(2) d 2 \\
& +\frac{1}{2} \sum_{\tau \rho} \int \frac{\delta^{2} A_{s, \mu}(1)}{\delta A_{\tau}(2) \delta A_{\rho}(3)} \delta A_{\tau}(2) \delta A_{\rho}(3) d 2 d 3,
\end{aligned}
$$

where for the second order derivative we have

$$
\begin{aligned}
& \frac{\delta^{2} A_{s, \mu}(1)}{\delta A_{\tau}(2) \delta A_{\rho}(3)} \\
& =\sum_{\zeta \eta} \int d 4 d 5 \frac{\delta^{2} A_{s, \mu}(1)}{\delta j_{\zeta}(4) \delta j_{\eta}(5)} \frac{\delta j_{\zeta}(4)}{\delta A_{\tau}(2)} \frac{\delta j_{\eta}(5)}{\delta A_{\rho}(3)} \\
& \quad+\sum_{\zeta} \int d 4 \frac{\delta A_{s, \mu}(1)}{\delta j_{\zeta}(4)} \frac{\delta^{2} j_{\zeta}(4)}{\delta A_{\tau}(2) \delta A_{\rho}(3)} \\
& =\sum_{\zeta \eta} \int d 4 d 5 g_{\mathrm{xc}, \mu \zeta \eta}(1,4,5) \chi_{\zeta \tau}(4,2) \chi_{\eta \rho}(5,3) \\
& \quad+\sum_{\zeta} \int d 4 f_{\mathrm{Hxc}, \mu \zeta}(1,4) \chi_{\zeta \mu \tau}^{(2)}(4,2,3)
\end{aligned}
$$

By inserting this back into Eq. (30) and then into Eq. (29) we obtain diagrams with the same structure as the last two diagrams of Fig. 7 but now the exact response function appears. Replacing the exact $\chi$ with $\chi_{s}$ we see that the diagram with $g_{\mathrm{xc}}$ cancels out whereas the diagram with $f_{\mathrm{xc}}$ is halved. As Eq. (31) contains $f_{\mathrm{Hxc}}=v+f_{\mathrm{xc}}$ we also get a diagram like the last diagram of Fig. 7 in which $f_{\mathrm{xc}}$ is replaced by the interaction $v$. In the many-body treatment this term arises from the expansion of $\mathbf{j}^{(1)}(1)$ too.

Let us continue our analysis of the nonvanishing diagrams for the photocurrent outside the sample. From the example of Fig. 8, we see that the functional derivative of $Q$ yields diagrams with interaction lines connecting different legs of the triangle. If we insert these diagrams back into Fig. 7 and then into Fig. 2 we recover the expansion at the exchange-only level of the photocurrent (see Fig. 1 with $W \rightarrow v$ ) provided we use $\chi_{s}$ instead of $\chi$ (this is justified since the expansion is first order in the interaction). The second and third diagrams of Fig. 2 are produced by a change in the Hartree field and are also naturally included in a lowest order many-body expansion in the bare interaction. If we want to compare the many-body diagrams of Fig. 1 where the interaction is screened then we also need an approximation to $f_{\mathrm{xc}}$ in terms of $W$. To lowest order in $W$ this approximation can be derived from the GW self-energy

$$
\Sigma_{\mathrm{xc}}(1,2)=-i G_{s}(1,2) W(1,2),
$$

where the screened interaction $W$ is the solution of

$$
W(1,2)=v(1,2)+\int d 3 d 4 v(1,3) P(3,4) W(4,2)
$$

with polarizability $P$ given by

$$
P(1,2)=-i G_{s}(1,2) G_{s}(2,1) .
$$

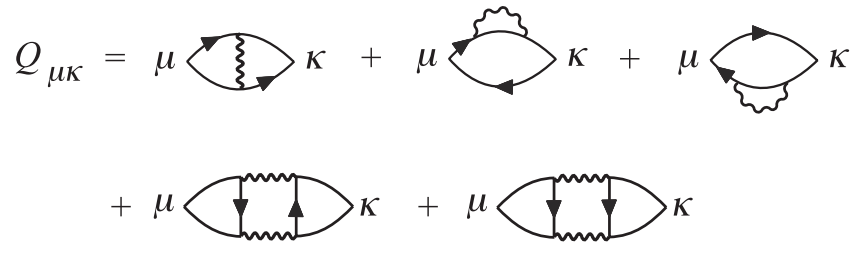

FIG. 9. Diagrams for $Q_{\mu \kappa}$ in a $G W$-type approximation for $f_{\mathrm{xc}}$. The wiggly lines denote screened interactions.

The corresponding equation for $Q$ is illustrated diagrammatically in Fig. 9. Such an expression was studied in Ref. 29 from a Luttinger-Ward functional. ${ }^{13,20}$ The diagrams also include two terms which are second order in $W$ and are important to include if one insists on having a conserving approximation. ${ }^{29}$ It assures, for instance, that the $f_{\mathrm{xc}}$ satisfies the linearized zero-force theorem ${ }^{38-41}$ which states that the exchange-correlation fields do not exert a net force on the system. By a differentiation of the corresponding function $Q$ and integration with the external fields we obtain the diagrams that contribute to the photocurrent. These are displayed in Fig. 10 and have the same structure as in Fig. 1. We recognize all diagrams (a)-(f) of this figure. The only difference is that we here still integrate over the two branches of the Keldysh contour. We also note that we have some diagrams with selfenergy insertions. This is because we still expand in terms of Kohn-Sham Green's functions rather than the fully dressed ones. The diagrams (g) and (h) of Fig. 1 which describe the change in the effective Hartree field are not included in Fig. 10 since they are already absorbed in the Hartree part $f_{\mathrm{H}}$ of $f_{\mathrm{Hxc}}$ and are represented by the second and third diagrams after the equal sign in Fig. 2. The remaining diagrams in Fig. 10 describe processes that are higher order in $W$. Such diagrams would also appear in a many-body treatment if we had expanded to higher order in the screened interactions. In photo-emission from metallic systems they would, for instance, describe processes in which there are multiple excitations of plasmons present. ${ }^{42,43}$ We have seen that within TDCDFT we can make a clear connection between the

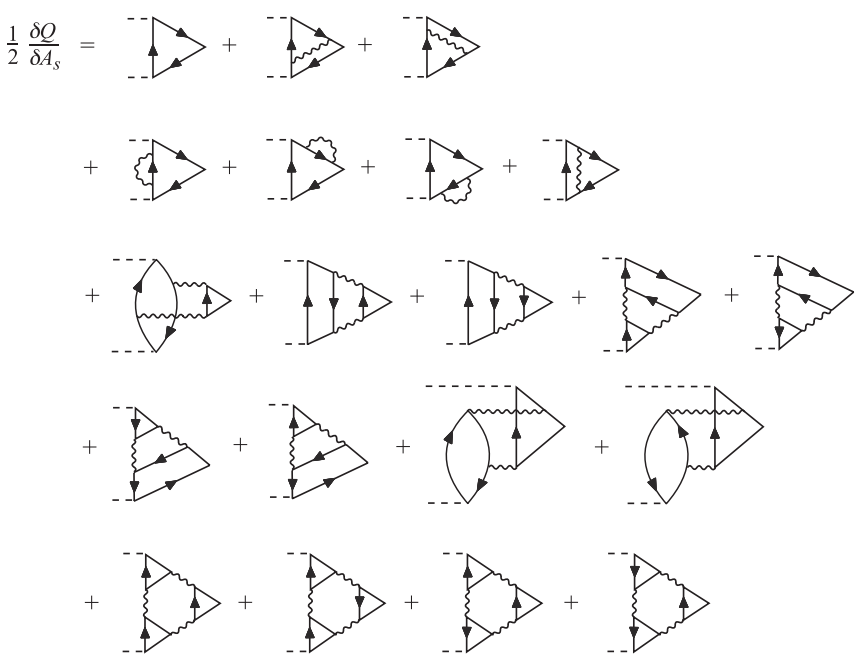

FIG. 10. Diagrams for the photocurrent derived from a $G W$-type approximation for $f_{\mathrm{xc}}$. The wiggly lines describe screened interactions. 
many-body expansion for the photocurrent and the KohnSham expression for it. The question that remains to be answered is whether knowledge of the photocurrent provides us with enough information to calculate the kinetic energy distribution of the photo-electrons. This question will be addressed in Sec. III E.

\section{E. Ultra-nonlocality}

In the transition from Eqs. (14) to (15) we made a conceptual step. The total angular distribution of the photocurrent was written as an integral over separate contributions from the electron kinetic energies $\epsilon$. This step requires a physical interpretation since it is not justified from a mathematical point of view. The expression can, however, be derived alternatively from Fermi's Golden Rule applied to the many-body system ${ }^{5}$ which amounts to a calculation of transition rates between many-body states. We could apply the same procedure to the Kohn-Sham system but then we would calculate transitions between Kohn-Sham Slater determinant states rather than between physical states. The corresponding formula would be given by removal of the integral sign in Eq. (22) after the substitution $\omega=\epsilon-\Omega$. This gives

$$
\frac{\partial^{2} J}{\partial \bar{\Omega} \partial \epsilon}(\hat{\mathbf{r}})=\frac{\sqrt{2 \epsilon}}{(2 \pi)^{3}}\left\langle\varphi_{s, \mathbf{q}}\left|\hat{w}_{s}^{\dagger} \hat{\mathcal{A}}_{s}(\epsilon-\Omega) \hat{w}_{s}\right| \varphi_{s, \mathbf{q}}\right\rangle .
$$

It is not difficult to see that we would run into a contradiction if we assumed that the right hand side of this equation would be identical to the right hand side of Eq. (15). This becomes clearer when we insert in Eq. (33) the explicit form of the Kohn-Sham spectral function of Eq. (23),

$$
\frac{\partial^{2} J}{\partial \bar{\Omega} \partial \epsilon}(\hat{\mathbf{r}})=\frac{\sqrt{2 \epsilon}}{(2 \pi)^{2}} \sum_{\epsilon_{j} \leq \mu}\left|\left\langle\phi_{j}\left|\hat{w}_{s}\right| \varphi_{s, \mathbf{q}}\right\rangle\right|^{2} \delta\left(\epsilon-\epsilon_{j}-\Omega\right) .
$$

If we took the example of a finite system then the spectrum on the right hand side of the equation would only have peaks at the Kohn-Sham energies, whereas the expression (15) has peaks at the true removal energies of the system. We conclude that Eq. (34) is not the same as Eq. (15) but that only the integrals over these functions up to $\epsilon=\mu+\Omega$ are the same. While this is apparent for a finite system for an infinite system the spectral peaks merge into a continuum and then it is not immediately obvious that the two expressions are different. However, there is no reason to assume that they are equal as the interpretation based on Fermi's Golden Rule demonstrates. We, therefore, conclude that the kinetic energy distribution cannot be directly calculated from knowledge of the current-density. This is mathematically clear since the momentum distribution requires knowledge of the one-particle density matrix which is no simple functional of the current density. However, in the experiment the kinetic energy is, in fact, measured by measuring the current at various positions in the detector. This is done by deflecting the photo-electron current with an applied electric or magnetic field, ${ }^{3}$ as depicted graphically in Fig. 11. Here, we display the detection of different kinetic energy components in the current. To every position in the detector plate there is assigned a corresponding kinetic energy. This detection process could be modeled in

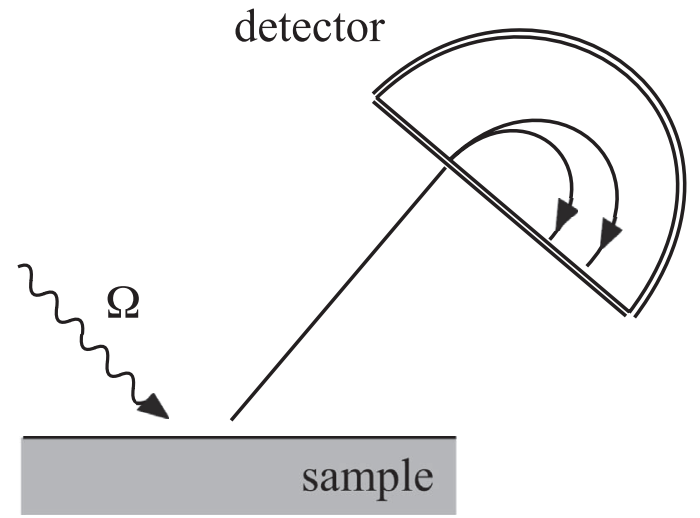

FIG. 11. Deflection of two different kinetic energy components of the current by a field in the detector.

TDCDFT as well. There exists an effective Kohn-Sham field $\mathbf{A}_{s}$ in the region of the detector which would bend the path of the currents in exactly the same way as the true electromagnetic field in the detector. Therefore, these kinetic energies could, in principle, also be measured in a Kohn-Sham approach. However, we realize that such a field must have knowledge of the true many-body spectral function in the sample in order to split the current in exactly the right way to produce peaks in the kinetic energy spectrum where the Kohn-Sham system has none. This means that the exchangecorrelation field in the detector far away from the sample (in fact, at a macroscopic distance in a real experiment) must depend in a nontrivial way on the many-body correlations in the sample. This is another illustration of extreme nonlocality of the exchange-correlation field for which we can find several other instances in density-functional theory. Other examples are the step structures in charge transfer processes in molecules, ${ }^{32,44,45}$ the macroscopic exchange-correlation field of molecular chains ${ }^{46}$ and the leaddependence of the exchange-correlation potential in quantum transport. ${ }^{47-49}$

\section{CONCLUSIONS}

We derived an exact expression within TDCDFT for the photocurrent of photo-emission spectroscopy. This expression involves an integral over the Kohn-Sham spectral function weighted with effective Kohn-Sham one-body interactions. Although this expression directly gives the angular dependence of the photocurrent it does not provide us directly with the kinetic energy distribution of the photo-electrons. This information can be obtained from TDCDFT as well, but there is a price to be paid for this. In order to do it we need to split the photocurrent into various kinetic energy distributions using an external exchange-correlation field outside the sample which depends in a very nonlocal manner on the many-body states inside the sample.

From a practical point of view we may wonder whether the derived expression of Eq. (34) could represent a sufficiently accurate, albeit non-exact, approximation to the kinetic energy distribution of the photo-electron spectrum. This probably depends highly on the studied system in question. 
For instance, for photo-emission of metallic systems the plasmon excitations are an important physical ingredient. Diagrammatically, these plasmonic effects are incorporated well in terms of Green's functions based on the GW approximation. It may well be that an xc-kernel based on a ShamSchlüter scheme at this level would give the required features in the photo-electron spectrum. These features then would come out, not by creating extra levels in the spectral functions, but by a redistribution of the intensities of the bare KohnSham spectral function by the matrix elements involving the xc-kernel. The most difficult case for TDCDFT is may be provided by finite systems, such as molecules, in which nontrivial doubly or multiple excited states ${ }^{50,51}$ may contribute important features to the spectral function.

\section{ACKNOWLEDGMENTS}

A.M.U. would like to thank the Alfred Kordelin Foundation for support. R.v.L. would like to thank the Academy of Finland for support. G.S. acknowledges funding by MIUR FIRB Grant No. RBFR12SW0J and financial support through travel grants Psi-K2 5813 of the European Science Foundation (ESF).

\section{APPENDIX: DERIVATION OF EQ. (10)}

In this appendix, we will give a derivation of Eq. (10). We define the retarded Green's function $\hat{\mathcal{G}}_{0}^{R}$ for a free particle outside the sample as

$$
\left(i \partial_{t}-\hat{t}\right) \hat{\mathcal{G}}_{0}^{R}\left(t, t^{\prime}\right)=\delta\left(t-t^{\prime}\right)
$$

where $\hat{t}$ is the kinetic energy of a free particle. The retarded Green's function of the sample satisfies

$$
\left(i \partial_{t}-\hat{h}\right) \hat{\mathcal{G}}^{R}\left(t, t^{\prime}\right)=\delta\left(t-t^{\prime}\right)+\int d \bar{t} \hat{\Sigma}^{R}(t, \bar{t}) \hat{\mathcal{G}}^{R}\left(\bar{t}, t^{\prime}\right)
$$

where $\hat{h}=\hat{t}+\hat{v}$ where $\hat{v}$ is the confining potential for the electrons in the sample (the potential due to the atomic nuclei) and $\hat{\Sigma}^{R}$ is the retarded many-body self-energy. Then we can write the Green's function of the sample in Dyson form as

$$
\hat{\mathcal{G}}^{R}=\hat{\mathcal{G}}_{0}^{R}+\hat{\mathcal{G}}_{0}^{R}\left(\hat{v}+\hat{\Sigma}^{R}\right) \hat{\mathcal{G}}^{R},
$$

where integrations over internal time variables are implied. If we now define the retarded $T$-matrix $\hat{T}^{R}$ by

$$
\hat{T}^{R}=\hat{v}+\hat{\Sigma}^{R}+\left(\hat{v}+\hat{\Sigma}^{R}\right) \hat{\mathcal{G}}_{0}^{R} \hat{T}^{R},
$$

then we can write

$$
\hat{\mathcal{G}}^{R}=\hat{\mathcal{G}}_{0}^{R}+\hat{\mathcal{G}}_{0}^{R} \hat{T}^{R} \hat{\mathcal{G}}_{0}^{R} .
$$

If we introduce the short notations

$$
\begin{aligned}
& \hat{X}_{\eta}^{R}=\left(1+\hat{T}^{R} \hat{\mathcal{G}}_{0}^{R}\right)(\omega+\eta \Omega), \\
& \hat{X}_{\eta}^{A}=\left(1+\hat{\mathcal{G}}_{0}^{A} \hat{T}^{A}\right)(\omega+\eta \Omega),
\end{aligned}
$$

then we can rewrite Eq. (9) as

$$
\begin{aligned}
G^{(2)<} & \left(\mathbf{x} t, \mathbf{x}^{\prime} t\right) \\
= & \sum_{\rho, \eta= \pm} e^{-i(\eta+\rho) \Omega t} \int \frac{d \omega}{2 \pi} \int d \mathbf{y} d \mathbf{y}^{\prime} \\
& \times\left\langle\mathbf{x}\left|\hat{\mathcal{G}}_{0}^{R}(\omega+\eta \Omega)\right| \mathbf{y}\right\rangle\left\langle\mathbf{y}\left|\hat{X}_{\eta}^{R} \hat{w}_{\eta} \hat{\mathcal{G}}^{<}(\omega) \hat{w}_{\rho} \hat{X}_{-\rho}^{A}\right| \mathbf{y}^{\prime}\right\rangle \\
& \times\left\langle\mathbf{y}^{\prime}\left|\hat{\mathcal{G}}_{0}^{A}(\omega-\rho \Omega)\right| \mathbf{x}^{\prime}\right\rangle .
\end{aligned}
$$

Now the matrix element of $\hat{\mathcal{G}}_{0}^{R}$ has the explicit form

$$
\left\langle\mathbf{x}\left|\hat{\mathcal{G}}_{0}^{R}(\nu)\right| \mathbf{y}\right\rangle=-\frac{\delta_{\sigma \sigma^{\prime}}}{2 \pi}\left\{\begin{array}{c}
\frac{e^{i \sqrt{2 v} r}}{r} v>0 \\
\frac{e^{-\sqrt{-2 v} r}}{r} v<0
\end{array},\right.
$$

where we defined $r=\left|\mathbf{r}-\mathbf{r}_{1}\right|$ with $\mathbf{x}=\mathbf{r}, \sigma$ and $\mathbf{y}=\mathbf{r}_{1}, \sigma^{\prime}$. Since $\hat{\mathcal{G}}^{<}(\omega)$ has only contributions for $\omega \leq \mu$ and $\Omega>0$ we see that this matrix element only gives a contribution for $r \rightarrow \infty$ when the argument of $\hat{\mathcal{G}}^{R}$ in Eq. (A1) is $\omega+\Omega$. This implies that the integral becomes

$$
\begin{aligned}
G^{(2)<} & \left(\mathbf{x} t, \mathbf{x}^{\prime} t\right) \\
= & \frac{1}{4 \pi^{2}} \int \frac{d \omega}{2 \pi} \int d \mathbf{r} d \mathbf{r}_{2} \frac{e^{i q\left|\mathbf{r}-\mathbf{r}_{1}\right|}}{\left|\mathbf{r}-\mathbf{r}_{1}\right|} \\
& \times\left\langle\mathbf{r}_{1}, \sigma\left|\hat{X}_{1}^{R} \hat{w}_{1} \hat{\mathcal{G}}^{<}(\omega) \hat{w}_{-1} \hat{X}_{1}^{A}\right| \mathbf{r}_{2}, \sigma^{\prime}\right\rangle \frac{e^{-i q\left|\mathbf{r}-\mathbf{r}_{2}\right|}}{\left|\mathbf{r}-\mathbf{r}_{2}\right|}
\end{aligned}
$$

where we defined $q>0$ by the relation $q^{2} / 2=\omega+\Omega$. If we are looking at point $\mathbf{r}$ far from the sample then we can use the approximation

$$
\frac{e^{i q\left|\mathbf{r}-\mathbf{r}_{1}\right|}}{\left|\mathbf{r}-\mathbf{r}_{1}\right|} \approx \frac{e^{i q(|\mathbf{r}|-\hat{\mathbf{r}} \cdot \mathbf{r})}}{|\mathbf{r}|}
$$

If we define $\mathbf{q}=q \hat{\mathbf{r}}$ and the plane wave state $|\mathbf{q}, \sigma\rangle$ with $\left\langle\mathbf{r}, \sigma \mid \mathbf{q}, \sigma^{\prime}\right\rangle=\delta_{\sigma \sigma^{\prime}} e^{i \mathbf{q} \cdot \mathbf{r}}$ then we can write

$$
\begin{aligned}
G^{(2)<}\left(\mathbf{x} t, \mathbf{x}^{\prime} t\right)= & \frac{1}{4 \pi^{2}} \frac{\delta_{\sigma \sigma^{\prime}}}{|\mathbf{r}|\left|\mathbf{r}^{\prime}\right|} \int \frac{d \omega}{2 \pi} e^{i q\left(|\mathbf{r}|-\left|\mathbf{r}^{\prime}\right|\right)} \\
& \times\left\langle q \hat{\mathbf{r}}, \sigma\left|\hat{X}_{1}^{R} \hat{w} \hat{\mathcal{G}}^{<}(\omega) \hat{w}^{\dagger} \hat{X}_{1}^{A}\right| q \hat{\mathbf{r}}^{\prime}, \sigma\right\rangle,
\end{aligned}
$$

where we used that the Green's function must be diagonal in the spin indices. If we then further define the state

$$
\left|\varphi_{q \hat{\mathbf{r}}}\right\rangle=\hat{X}_{1}^{A}|q \hat{\mathbf{r}}, \sigma\rangle=\left(1+\hat{\mathcal{G}}_{0}^{A} \hat{T}^{A}\right)(\omega+\Omega)|q \hat{\mathbf{r}}, \sigma\rangle,
$$

then the desired Eq. (10) follows immediately from Eq. (A3). It remains to give a more explicit characterization of the state $\left|\varphi_{q \hat{\mathbf{r}}}\right\rangle$. It satisfies the equation

$$
\left|\varphi_{q \hat{\mathbf{r}}}\right\rangle=|\mathbf{q}, \sigma\rangle+\frac{\hat{v}+\hat{\Sigma}(\omega+\Omega)}{\omega+\Omega-\hat{t}-i \eta}\left|\varphi_{q \hat{\mathbf{r}}}\right\rangle,
$$

which represents an advanced solution of the LippmannSchwinger equation with incoming plane wave boundary conditions. Equivalently, we can write Eq. (A4) as

$$
\left[\hat{h}+\hat{\Sigma}^{A}\left(\frac{q^{2}}{2}\right)\right]\left|\varphi_{q \hat{\mathbf{r}}}\right\rangle=\frac{q^{2}}{2}\left|\varphi_{q \hat{\mathbf{r}}}\right\rangle
$$

and we see that it equivalently satisfies a quasi-particle type 
equation for a continuum state. We have recovered exactly Eq. (11).

${ }^{1}$ A. Einstein, Ann. Phys. 322, 132 (1905).

${ }^{2}$ N. D. Lang and W. Kohn, Phys. Rev. B 3, 1215 (1971).

${ }^{3}$ A. Damascelli, Z. Hussain, and Z.-X. Shen, Rev. Mod. Phys. 75, 473 (2003).

${ }^{4}$ C.-O. Almbladh, Phys. Scr. 32, 341 (1985).

${ }^{5}$ C.-O. Almbladh and L. Hedin, in Handbook on Synchrotron Radiation, edited by E. E. Koch (North-Holland Publishing, 1983), Vol. 1, p. 607.

${ }^{6}$ C.-O. Almbladh, J. Phys.: Conf. Ser. 35, 127 (2006).

${ }^{7}$ W. Bardyszewski and L. Hedin, Phys. Scr. 32, 439 (1985).

${ }^{8}$ R. M. Dreizler and E. K. U. Gross, Density Functional Theory: An Approach to the Quantum Many-Body Problem (Springer, Berlin, 1990).

${ }^{9}$ E. Engel and R. M. Dreizler, Density Functional Theory: An Advanced Course (Springer, Heidelberg, 2011).

${ }^{10}$ U. von Barth, "Basic density-functional theory-An overview," Phys. Scr. T109, 9 (2004).

${ }^{11}$ C. A. Ullrich, Time-Dependent Density-Functional Theory: Concepts and Applications (Oxford University Press, Oxford, 2012).

${ }^{12}$ R. van Leeuwen, Int. J. Mod. Phys. B 15, 1969 (2001).

${ }^{13}$ R. van Leeuwen, N. E. Dahlen, G. Stefanucci, C.-O. Almbladh, and U. von Barth, Lecture Notes in Physics (Springer, 2006), Vol. 706, p. 33.

${ }^{14}$ C. A. Ullrich and Z. Yang, Braz. J. Phys. 44, 154 (2014).

${ }^{15}$ M. Ruggenthaler and R. van Leeuwen, EPL 95, 13001 (2011).

${ }^{16}$ G. Vignale and W. Kohn, Phys. Rev. Lett. 77, 2037 (1996).

${ }^{17}$ G. Vignale, Phys. Rev. B 70, 201102(R) (2004).

${ }^{18}$ I. V. Tokatly, Phys. Rev. B 83, 035127 (2011).

${ }^{19}$ H. Husser, J. van Heys, and E. Pehlke, Phys. Rev. B 84, 235135 (2011).

${ }^{20} \mathrm{G}$. Stefanucci and R. van Leeuwen, Nonequilibrium Many-Body Theory of Quantum Systems (Cambridge University Press, 2013).

${ }^{21}$ R. van Leeuwen, Prog. Theor. Chem. Phys. 14, 43-68 (2003).

${ }^{22} \mathrm{R}$. van Leeuwen, in Progress in Nonequilibrium Green's Functions II, edited by M. Bonitz and D. Semkat (World Scientific, Singapore, 2003), p. 427.

${ }^{23}$ C.-O. Almbladh and U. von Barth, Phys. Rev. B 31, 3231 (1985).

${ }^{24}$ R. Fukuda, M. Komachiya, S. Yokojima, Y. Suzuki, K. Okumura, and T. Inagaki, Prog. Theor. Phys. Suppl. 121, 1-428 (1995).

${ }^{25} \mathrm{M}$. Valiev and G. W. Fernando, e-print arXiv:cond-mat/9702247.
${ }^{26}$ I. V. Tokatly and O. Pankratov, Phys. Rev. Lett. 86, 2078 (2001).

${ }^{27}$ I. V. Tokatly, R. Stubner, and O. Pankratov, Phys. Rev. B 65, 113107 (2002).

${ }^{28}$ L. Reining, V. Olevano, A. Rubio, and G. Onida, Phys. Rev. Lett. 88, 066404 (2002).

${ }^{29}$ U. von Barth, N. E. Dahlen, R. van Leeuwen, and G. Stefanucci, Phys. Rev. B 72, 235109 (2005).

${ }^{30}$ M. Hellgren and U. von Barth, Phys. Rev. B 78, 115107 (2008).

${ }^{31}$ M. Hellgren and U. von Barth, J. Chem. Phys. 131, 044110 (2009).

${ }^{32}$ M. Hellgren and E. K. U. Gross, Phys. Rev. A 88, 052507 (2013).

${ }^{33}$ In principle $F_{\mathrm{xc}}$ also have an explicit dependence on the xc-field $\mathbf{A}_{\mathrm{xc}}$. However, this is not the case for the approximations we consider.

${ }^{34}$ L. J. Sham and M. Schlüter, Phys. Rev. Lett. 51, 1888 (1983).

${ }^{35}$ R. van Leeuwen, Phys. Rev. Lett. 76, 3610 (1996).

${ }^{36}$ D. Mearns and W. Kohn, Phys. Rev. A 35, 4796 (1987).

${ }^{37}$ In contrast the Laplace transform of the response function is invertible, see Ref. 12.

${ }^{38}$ J. F. Dobson, Phys. Rev. Lett. 73, 2244 (1994).

${ }^{39}$ G. Vignale, Phys. Rev. Lett. 74, 3233 (1995).

${ }^{40}$ G. Vignale, Phys. Lett. A 209, 206 (1995).

${ }^{41}$ M. Mundt, S. Kümmel, R. van Leeuwen, and P.-G. Reinhard, Phys. Rev. A 75, 050501(R) (2007).

${ }^{42}$ F. Aryasetiawan, L. Hedin, and K. Karlsson, Phys. Rev. Lett. 77, 2268 (1996).

${ }^{43}$ M. Guzzo, J. J. Kas, F. Sottile, M. G. Silly, F. Sirotti, J. J. Rehr, and L. Reining, Eur. Phys. J. B 85, 324 (2012).

${ }^{44}$ P. Elliott, J. I. Fuks, A. Rubio, and N. T. Maitra, Phys. Rev. Lett. 109,266404 (2012).

${ }^{45}$ J. I. Fuks, P. Elliott, A. Rubio, and N. T. Maitra, J. Phys. Chem. Lett. 4, 735 (2013).

${ }^{46}$ M. van Faassen, P. L. de Boeij, R. van Leeuwen, J. A. Berger, and J. G. Snijders, Phys. Rev. Lett. 88, 186401 (2002).

${ }^{47}$ A.-M. Uimonen, E. Khosravi, A. Stan, G. Stefanucci, S. Kurth, R. van Leeuwen, and E. K. U. Gross, Phys. Rev. B 84, 115103 (2011).

${ }^{48}$ S. Kurth and G. Stefanucci, Phys. Rev. Lett. 111, 030601 (2013).

${ }^{49}$ P. Schmitteckert, M. Dzierzawa, and P. Schwab, Phys. Chem. Chem. Phys. 15, 5477 (2013).

${ }^{50}$ P. Elliott, S. Goldson, C. Canahui, and N. T. Maitra, Chem. Phys. 391, 110 (2011).

${ }^{51}$ M. Ruggenthaler, S. E. B. Nielsen, and R. van Leeuwen, Phys. Rev. A 88, 022512 (2013). 